\title{
Eclipse mapping of Algol-type systems with oscillating $\delta$ Scuti type components
}

\author{
Patricia Lampens ${ }^{1}$, Lore Vermeylen ${ }^{1}$, Peter De Cat ${ }^{1}$, Paul Van Cauteren ${ }^{2}$ \\ ${ }^{1}$ Royal Observatory of Belgium (ROB), Ringlaan 3, 1180 Brussels, Belgium \\ ${ }^{2}$ Humain station, Royal Observatory of Belgium, Ringlaan 3, 1180 Brussels, Belgium
}

\begin{abstract}
The oscillating Algol-type (oEA) stars are the former secondaries of evolved, semi-detached eclipsing binaries which are (still) undergoing mass transfer and form a newly detected class of pulsators close to the main sequence. Their unique feature consists of mass accretion onto the atmosphere of the pulsating star. Mass accretion affects the mass, radius, density as well as the star's evolution. Such stars are therefore very attractive targets for asteroseismic studies.

A new tool for mode identification of pulsating components in eclipsing binaries consists in using the event of primary eclipse as a spatial filter to resolve the pulsations across the stellar surface of the pulsator. During an eclipse, depending on the spatial structure of modes represented by the wave numbers $(l, m)$, different shapes of the pulsation amplitude and phase variations can be observed. A comparison between the modelled and the observed amplitudes and phases then provides the correct mode identification. The method needs a geometrical model, the pulsation frequencies and the light curves as input.

The geometrical model can be obtained from a simultaneous modelling of the light and radial velocity curves. The pulsation frequencies are obtained from subtracting a binary model from the original light curves followed by a frequency analysis of the residuals. We will identify and illustrate systems where observations with a fast CCD camera equipping the 3.6-m DOT telescope could deliver the data needed for the application of the eclipse mapping method.
\end{abstract}

Keywords: Stars: eclipsing binary - Stars: fundamental parameters - Stars: accretion - Stars: Delta Scuti

\section{Introduction}

The purpose of asteroseismology is to understand the physics of pulsation in order to probe the stellar interiors of particular stars. Though the excitation mechanism is well-understood for $\delta$ Scuti stars, namely the $\kappa$-mechanism active in the partial ionisation zones of H I, He I and He II (see Pamyatnykh 2000; Antoci et al.2014), so far, unknown amplitude limiting and mode selection mechanisms are operating in these pulsators. Only a fraction of the theoretically predicted modes are observed and this results in (too) many free parameters in the pulsation models. It is therefore essential to gain additional information on the fundamental stellar properties of the pulsator in an independent way. One way is to study a pulsating star in a binary system in much detail, since binary systems with well-characterized components provide stringent constraints for both evolutionary and asteroseismic modelling. Detached and semi-detached Eclipsing Binary Systems (EBs) are particularly well-suited as their detailed observation enables to derive the physical parameters (masses, radii and luminosities) 
of both components. EBs furthermore provide the component's effective temperatures, as well as the distance, if stellar atmosphere models are used (Maceroni 2006).

About sixty to seventy percent of all stars of the Solar neighbourhood are members of binary or multiple systems (i.e. 56\% for F(7)-M stars (for log Period < 3.61), Eggenberger et al. 2004; 75\% for O-B stars, Mason et al. 2001). Yet, these facts are usually ignored in the study of stellar pulsation. However, there is clear observational evidence that duplicity also affects the pulsation properties in a growing number of cases (e.g. HD 177863, De Cat \& Aerts 2002; KOI 54, Welsch et al.2011; KIC 9851944, Guo et al. 2016; KIC 6048106, Samadi Ghadim et al. 2018).

\section{Eclipsing binary systems and their fundamental parameters}

Eclipsing systems are essential contributors of fundamental stellar parameters. These systems are (generally) close binaries which can also be observed as spectroscopic binaries (both single- and double-lined systems). The combined analysis of their photometric (light) and spectroscopic (radial velocity) curves allows the determination of the component masses and radii in a most straightforward way. Based on increasingly better data sets (e.g. the Kepler light curves) and high signal-tonoise spectroscopy, the determination of these fundamental parameters may reach accuracies of the order of a few percent (Matson et al.2016). Accurate masses and radii in turn allow to determine the component gravities precisely, whereas an accurate determination of the radii and the effective temperatures allows to derive the absolute bolometric component luminosities in an accurate way, e.g. the study of V578 Mon by Hensberge et al. (2000) who determined an accurate distance to the Rosette nebula. Another illustrative example is the study of the massive eclipsing binary system with accuracies better than $4 \%$ on the component masses and of the order of $1 \%$ on the component radii (Kourniotis et al.2015). In addition, the authors obtained the distance of the system (and the cluster it belongs to) with a precision of $2 \%$ by fitting the spectral energy distribution.

\section{The oEA stars}

The oEA stars are the former secondaries of evolved, semi-detached eclipsing systems which are (still) undergoing mass transfer and form a recently detected class of pulsators close to the main-sequence (Mkrtichian et al.2002). Their unique feature consists of mass accretion onto the atmosphere of the pulsating star. Mass accretion affects the mass, radius, density as well as the star's evolution (depending on the accretion rate).

A recent list of 118 eclipsing systems with $\mathrm{P}_{\text {orb }}<13 \mathrm{~d}$ containing both detached and semidetached systems has been compiled by Liakos \& Niarchos (2017). The pulsating components occupy the same region of the $\mathrm{H}-\mathrm{R}$ diagram as the classical $\delta$ Scuti stars, with masses ranging from 1.6 to 2.4 $\mathrm{M}_{\odot}$ and $\mathrm{T}_{\text {eff }}$ ranging from 7100 to $9500 \mathrm{~K}$ (Figs. 6 and 7 in Liakos \& Niarchos 2017) ${ }^{1}$. Concerning their pulsations, they seem to share the properties of the (supposed) single $\delta$ Scuti stars, which exhibit radial and non-radial pulsations with frequencies ranging between 3 and $80 \mathrm{~d}^{-1}$ (Breger 2000) and can oscillate in both $p$ and $g$ modes. However, their evolutionary history is very different and their envelopes are still accreting gas. Such stars are therefore very attractive targets for asteroseismic studies.

\footnotetext{
${ }^{1}$ In 1999, at the 6th Vienna Workshop in Astrophysics, only six cases of eclipsing binary systems with a pulsating component of $\delta$ Scuti type were known to Lampens \& Boffin (2000).
} 
Since they belong to the group of spectroscopic binaries with eclipses, their absolute stellar parameters can be determined with a high level of accuracy, e.g. using the code PHOEBE (PHysics Of Eclipsing BinariEs) based on the Wilson-Devinney method (Prša et al. 2011), e.g. CT Her (Lampens et al. 2011). Such information is crucial, not only for understanding the evolutionary status of systems in the slow-mass transfer stage (Mkrtichian et al.2007), but also for application of the method of spatial filtering presented below in view of a fine-tuning of the pulsation spectra (Mkrtichian et al. 2002).

\section{The method of Periodic Spatial Filtering (PSF)}

This new method of mode identification for pulsating components in EBs was first suggested by Nather \& Robinson (1974). The method uses the primary eclipse event as a spatial filter to resolve the pulsations across the stellar surface of the pulsator. It is based on the fact that during an eclipse of the pulsating component the occulting star acts as a screen with a timely variable shape. During an eclipse, depending on the spatial structure of the modes represented by the wave numbers $(l, m)$, different shapes of the pulsation amplitude and phase variations are observed. The comparison of the modelled and the observed amplitude and phase patterns provides the correct mode identification. The PSF method (or the alternative method of 'dynamic eclipse mapping', Bíró \& Nuspl 2011) needs a geometrical model, the pulsation frequencies as well as the light curves as input. Mode identification can be carried out by examining the changes during (partial or total) eclipse of the non-radial pulsator. An illustration of the 'screening' effect during a primary eclipse is provided in Fig. 1.

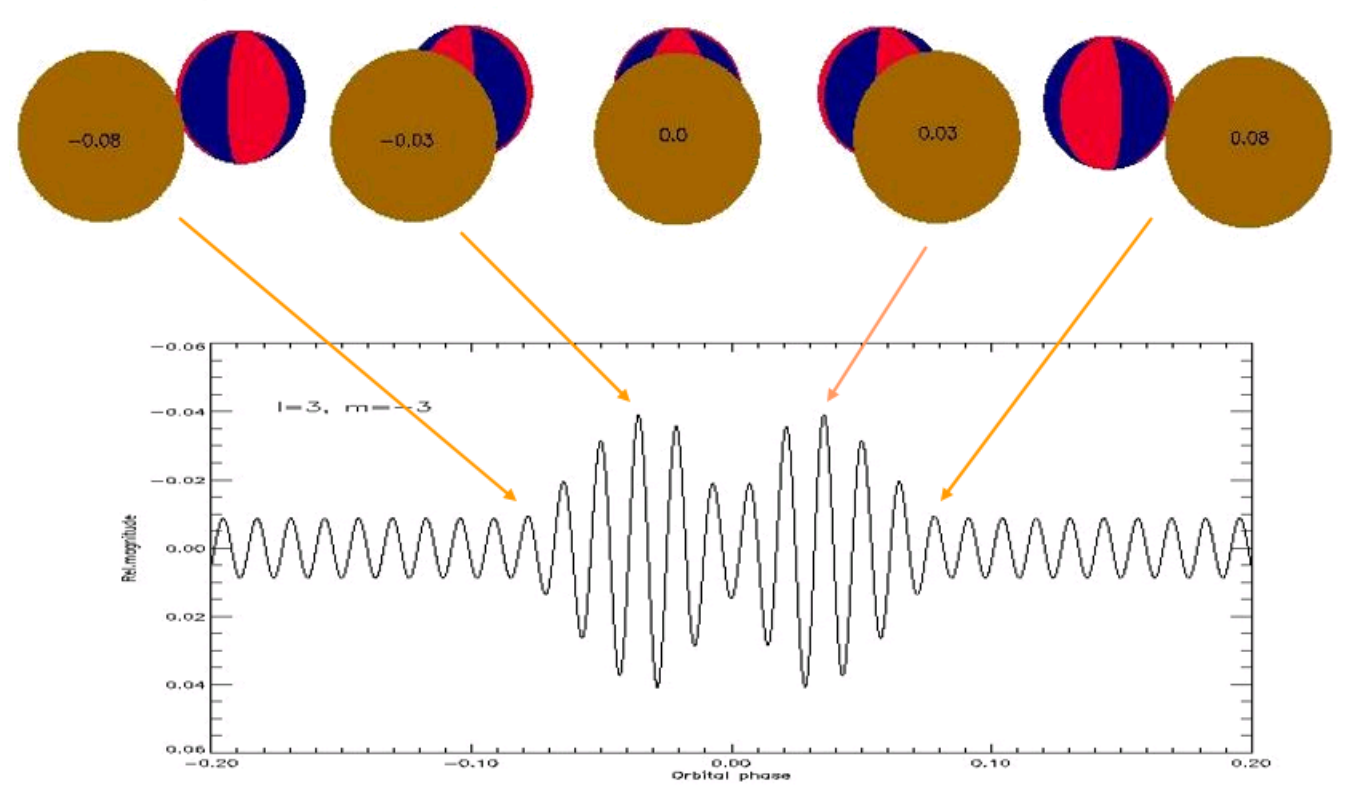

Figure 1: Illustration of the PSF method. The modified light curve was computed at the different phases of the partial primary eclipse for the prograde non-radial mode $l=3, m=-3$. Courtesy of D. Mkrtichian after A. Gamarova \& A. Hatzes.

The first attempt to perform mode identification of an oEA star during eclipse was done for RZ Cas by Gamarova et al. (2003). Later on, this method was applied to the oEA stars AB Cas (Rodríguez et al. 2004b) and RZ Cas (Rodríguez et al. 2004a). These authors reported preliminary mode identifications from the application of the PSF method to the ascending and descending branches of the primary 
minima of the light curves. Mkrtichian et al. (2018) revisited the existing photometric/spectroscopic data on RZ Cas and drew a detailed picture based on 9 years of monitoring between 1999 and 2009. The authors concluded that a rapid, intense mass transfer event had occurred in the period 2000-2001, leading to a fast change of its orbital period as well as a drastic decrease in the amplitude of the main pulsation frequency. They explain these facts as being due to cyclic variations of the oblateness of the secondary star coupled to a 9 yr cycle of magnetic activity. Application of the PSF method unto the residuals of two (partial) eclipse light curves enabled the identification of the tesseral $l=2, m=-1$ mode for the main frequency.

Also note that the method of 'dynamic eclipse mapping' was developed and tested using artificially generated data, with promising results. The method is - in principle - able to deal with many simultaneous modes provided that the orientation of the rotation axis is known. The first application on real data, however, proved to be challenging due to the regular presence of the orbital frequency spacing (this concerns KIC 10661783, Bíró 2013).

\section{Choice of instrument and future perspective}

The class of oEA stars offers opportunities to increase our knowledge of the pulsational properties in the presence of tides and mass accretion and provides another tool for mode identification of pulsating stars. The instrumental requirements of the PSF method must not be underestimated since we require that the data be obtained with a high cadence and high quality (high $\mathrm{S} / \mathrm{N}$ ) during the critical phase of the primary eclipse. Space observations (such as coming from CoRoT, Kepler/K2, or TESS (for the bright objects)) should be ideal, however most of the current space light curves have been obtained in the low cadence mode of $30 \mathrm{~min}$ which is obviously insufficient for this application. Combining multiple primary eclipse light curves to increase the time resolution may introduce biases since the (many) pulsation modes may not repeat exactly as a function of orbital phase.

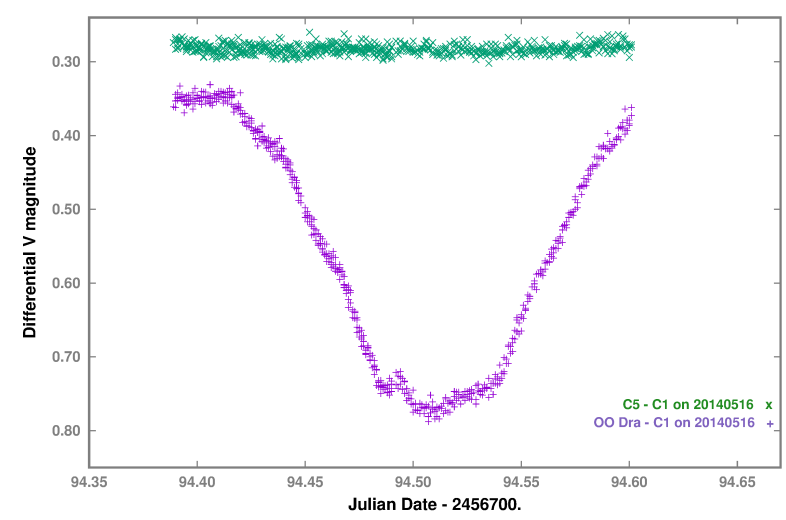

Figure 2: V-band light curve of OO Dra showing the phase of primary eclipse (project HOACS, year 2014). The presence of nonradial pulsation during this phase is obvious.

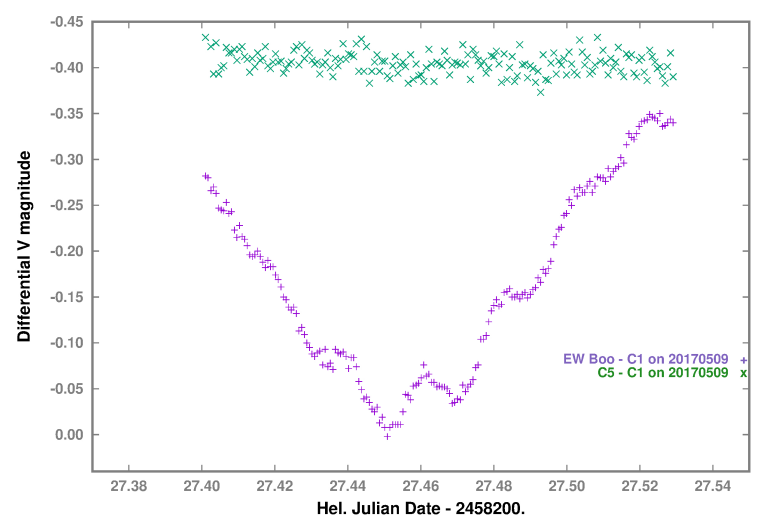

Figure 3: V-band light curve of EW Boo showing the phase of primary eclipse (project HOACS, year 2014). The presence of nonradial pulsation during this phase is obvious.

To illustrate our objective, we present the light curves of two potential targets which are showing on-going (non-)radial pulsation during the phase of the primary eclipse. We searched among the oEAs for which we dispose of light curves obtained from the project HOACS (operated by the ROB at the remote station of Humain, Belgium) and radial velocity curves acquired with the Mercator telescope equipped with the high-resolution HERMES spectrograph (Raskin et al.2011). Figs. 2 and 3 show 
the light curves obtained during the phase of primary eclipse for OO Dra (Zhang et al.2014) and EW Boo (Zhang et al. 2015), respectively. Their main pulsation periods are short (of order of 25-30 min). Therefore, we would need both high cadence and high $\mathrm{S} / \mathrm{N}$ (during the phase of the primary minimum). A medium-sized (large) telescope with a camera offering a cadence of less than $1 \mathrm{~min}$ under excellent atmospheric conditions would be well-adapted for this purpose, particularly if multicolour observations could also be performed. This would give the benefit of extracting additional information about the modes via the method of amplitude ratios and phase differences (Garrido 2000, Dupret et al. 2003). The CCD optical imager mounted at the Cassegrain focus of the 3.6-m Devasthal Optical Telescope (DOT) is equipped with several filters and can be operated with a minimum exposure time of $300 \mathrm{msec}$ (Pandey et al. 2018). The typical V magnitude of our (so far) selected targets during primary eclipse lies in the magnitude range 11 to 13 . For a $22^{\text {nd }}$ mag star, the signal in the $\mathrm{V}$-band may reach $\mathrm{S} / \mathrm{N} \sim 100$ with a $1 \mathrm{hr}$ exposure time (for a seeing of $\sim 1.1 \mathrm{arcsec}$ and a read-out noise of 2.5 electrons, Pandey et al.2018). Thus, we may adopt 1-2 sec exposure time for an $11^{\text {th }}$ mag star aiming to obtain $\mathrm{S} / \mathrm{N}=400$ (or longer exposure times if a neutral density filter would be available). Such an instrument may provide fast, high-precision time-series photometry of selected oEA stars during the essential phase of primary eclipses.

Looking for good candidates from $\mu$ mag accuracy space missions (e.g. CoRoT, Kepler, TESS or K2) would be the next option, since many new cases of semi-detached eclipsing binary systems with fainter oscillating primary components have been identified while analysing the space data. However, unless these light curves are accompanied by well-defined radial velocity curves, multi-epoch (preferably high-resolution) spectroscopy of these objects is fundamental to enable an accurate description of the binary model as required for the PSF and the 'dynamic eclipse mapping' method.

\section{Acknowledgements}

This research is supported by the Belgo-Indian Network for Astronomy \& Astrophysics (BINA), approved by the Belgian Federal Science Policy Office (BL/11/IN07, Govt. of Belgium) and the Department of Science and Technology (DST/INT/Belg/P-02, Govt. of India). We thank Dr Santosh Joshi (ARIES) for assistance regarding the (attempted) observations at the DOT. Furthermore, we gratefully acknowledge the support of the directors of the Royal Observatory of Belgium for purchasing and operating a small optical observatory at the radio-astronomy station of Humain under the project named HOACS (Humain Optical Observatory for Astrophysics of Coeval Stars).

\section{References}

Antoci V., Cunha M., Houdek G. et al. 2014, ApJ, 796, 118

Bíró I. B. 2013, EAS Publications Series, 64, 331

Bíró I. B., Nuspl J. I. 2011, MNRAS, 416, 1601

Breger M. 2000, ASP Conf. Ser., 210, 3

De Cat P., Aerts C. 2002, A\&A, 393, 965

Dupret M.-A., Scuflaire R., Noels A. et al. 2003, Ap\&SS, 284, 129

Eggenberger A., Halbwachs J.-L., Udry, S. et al. 2004, RMxAA Conf. Ser., 21, 28

Gamarova A. Y., Mkrtichian D. E., Rodriguez E. et al. 2003, ASP Conf. Ser., 292, 369

Garrido R. 2000, ASP Conf. Ser., 210, 67

Guo Z., Gies D. R., Matson, R. A. et al. 2016, ApJ, 826, 69

Hensberge H., Pavlovski K., Verschueren W. 2000, A\&A, 358, 553 
Kourniotis M., Bonanos A. Z., Williams S. J. et al. 2015, A\&A, 582, A42

Lampens P., Boffin H. M. J. 2000, ASP Conf. Ser., 210, 309

Lampens P., Strigachev, A., Kim, S.-L. et al. 2011, A\&A, 534, A111

Liakos A., Niarchos P. 2017, MNRAS, 465, 1181

Maceroni C. 2006, ASP Conf. Ser. 349, 41

Mason, B. D., Gies D. R., Hartkopf W. I. 2001, ApSSL, 264, 37

Matson R. A., Gies D. R., Guo Z. et al. 2016, AJ, 151, 139

Mkrtichian D. E., Kusakin A. V., Gamarova A. Y. et al. 2002, ASP Conf. Ser. 256, 259

Mkrtichian D. E., Kim S.-L., Rodríguez, E. et al. 2007, ASP Conf. Ser. 370, 194

Mkrtichian D. E., Lehmann H., Rodríguez E. et al. 2018, MNRAS, 475, 4745

Nather R. E., Robinson E. L. 1974, ApJ, 190, 637

Pamyatnykh A. A. 2000, ASP Conf. Ser., 210, 215

Pandey S. B., Yadav R. K. S., Nanjappa N. et al. 2018, BSRSL, 87, 25

Prša A., Matijevic G., Latkovic O. et al. 2011, ascl soft, soft06002P

Raskin G., Van Winckel H., Hensberge H. et al. 2011, A\&A, 526, A69

Rodríguez E., García J. M., Mkrtichian D. E. et al. 2004a, MNRAS, 347, 1317

Rodríguez E., García J. M., Gamarova A. Y. et al. 2004b, MNRAS, 353, 310

Samadi Ghadim A., Lampens P., Jassur D. M. 2018, AcA, 68, 425

Welsh W. F., Orosz J. A., Aerts C. et al. 2011, ApJS, 197, 4

Zhang X. B., Deng L. C., Tian J. F. et al. 2014, AJ, 148, 106

Zhang X. B., Luo Y. P., Wang K. et al. 2015, AJ, 149, 96 